\title{
Detection of Fatigue Cracks at Weld Toes by Crack Detection Paint and Surface SH Wave*
}

\author{
Ichihiko Takahashi and Michio Ushijima \\ National Maritime Research Institute, Tokyo 181-0004, Japan
}

A crack detection (CD) paint was applied to weld lines of a transverse rib welded joint specimen of a rolled steel for welded structures, and the effects of the paint on visual detection of fatigue cracks were evaluated by performing a fatigue test. Remarkable color development was observed in the CD paint when fatigue cracks propagated along the weld toe lines. At intervals in the fatigue test, surface SH (Secondary Horizontal) wave tests were also carried out in order to confirm the existence of fatigue cracks, and the test results were compared with the color development in the CD paint. [doi:10.2320/matertrans.I-MRA2007845]

(Received August 22, 2006; Accepted February 22, 2007; Published May 25, 2007)

Keywords: fatigue crack, welded joint, weld toe, visual inspection, crack detection paint, microcapsule, surface secondary horizontal (SH) wave

\section{Introduction}

Fatigue crack detection in the present ship hull inspection mostly depends on visual inspection. However, it is very difficult to detect fatigue cracks in actual ship structures because of several reasons, i.e. complicated structural details (such as welded joints), poor accessibility and severe environments, insufficient lighting, etc. A questionnaire survey on fatigue damage and maintenance of bulk carriers revealed that approximately $60 \%$ of fatigue cracks are found through the present inspection and the rest $40 \%$ are missed, and that $30 \%$ of the cracks grow longer than $200 \mathrm{~mm}$ and $10 \%$ of the cracks grow longer than $500 \mathrm{~mm}^{1)}$ Because such oversight of cracks possibly occur in other types of ships or other structures, i.e. tankers, passenger ships, steel bridges, etc., it is necessary for improvement of crack detectability to provide a new measure that can effectively support visual inspection on the spot.

On the one hand, research on a crack detection technique using a special paint called 'Smart Paint' had been carried out for about 10 years since 1990 at Lehigh University in the United States. ${ }^{2)}$ The 'Smart Paint' system consists of an ordinary epoxy resin maintenance paint and microcapsules containing a liquid dye, and the failure of the microcapsules due to crack initiation and propagation in the base metal and the paint causes a flow of the dye onto the paint surface, facilitating visual detection with the color development of the dye. Figure 1 illustrates the system. As compared with other non-destructive inspection techniques, e.g. an ultrasonic testing, a magnetic particle testing, etc., the primary merits and demerits of the crack detection paint system are as follows.

\section{Merits:}

- Any special equipment but for lighting is not required. Consequently special skills to operate such equipment are not required.

- It takes far shorter time to find a crack than other methods.

- Magnitude of damage can be intuitively grasped on the

*This Paper was Originally Published in Japanese in JOURNAL OF JSNDI 54 (2005) 503-508.

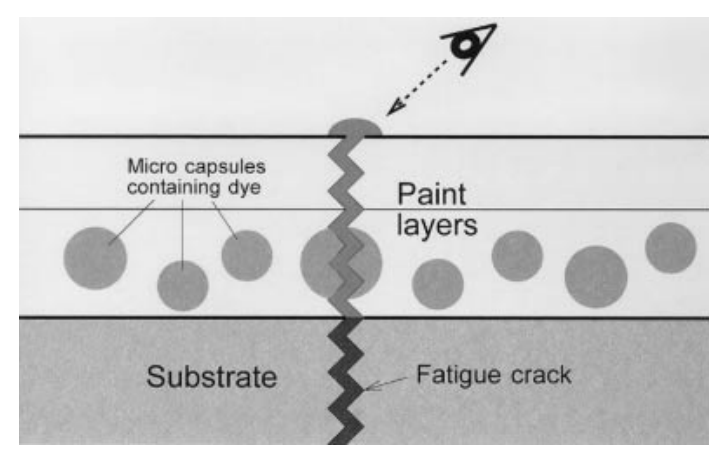

Fig. 1 Illustration of the smart paint system.

spot, because the location and length of a crack are roughly known at a glance. And any detailed marking for reconfirmation, which is ordinarily important for later maintenance or repair work, is not necessary.

- Cracks can be visually recognized from some distance. Therefore, remote inspection to inaccessible sites (e.g. elevated spots) is possible by using field glasses.

Demerits:

- Application of the crack detection paint takes time and labor for the surface preparation, painting and curing procedures, as in the case of ordinary painting work.

- The subject of inspection is limited to surface cracks.

- Because the crack height cannot be evaluated from the dye indication only, some other non-destructive testing techniques, by which the crack height can be quantitatively evaluated, should be used in combination in order to plan properly the subsequent maintenance or repair.

The authors have made a domestic pilot manufacturing of the crack detection (CD) paint based on the concept of the above 'Smart Paint' and basic performance evaluation of the paint by fatigue tests of both plate and welded joint specimens, proving the availability as a support tool for visual crack inspection. ${ }^{3)}$ Although rather short fatigue cracks (about 2 to $3 \mathrm{~mm}$ length) can be detected by the CD paint and the color development well agrees to the actual crack length, ${ }^{3)}$ some false color development may occur when the paint is damaged by external impact force or simple paint cracking of the under coat. Therefore it is desirable to 
confirm the existence of a crack in the base metal by some other means in combination when color development in the paint is found on the spot. Among various testing methods, the surface $\mathrm{SH}$ wave test is considered to be most suitable for such kind of confirmation test. The study of the surface SH wave test has become active recently, and the application to the surface cracks, which can be hardly detected by SV (Secondary Vertical) waves, is expected. The surface SH wave test has the advantages that the influence of the surface state is small because the direction of the wave vibration is parallel to the test surface, and that other waves of different mode do not coexist. ${ }^{4)}$ Moreover, it is considered as a great merit from a practical point of view that the surface $\mathrm{SH}$ wave can be applied when the accurate plate thickness and the structural details on the spot are unknown because it does not use the bottom echo. In addition, the recent improvement of the couplant for shear waves, which produced suitable viscosity for field work, has been encouraging the extensive application of the surface $\mathrm{SH}$ wave test to various kinds of on-the-spot inspections. ${ }^{4,5)}$

Because many of fatigue cracks in ship hull structures initiate and propagate along weld toes, simultaneous application of the CD paint and the surface $\mathrm{SH}$ wave test to actual weld toe cracks and comparison of the both test results are essential to evaluate the effectiveness of the combined inspection technique. In this study, a fatigue test was performed on a transverse rib welded joint specimen, to which the CD paint was applied beforehand. During the fatigue test, the color development in the $\mathrm{CD}$ paint was observed and the surface SH wave test was carried out in combination at intervals, verifying the effectiveness of the combined inspection technique for the weld toe cracks. The discussion of crack shape evaluation in this paper is mainly on locations and lengths of surface cracks, while the possibility of crack height evaluation by the surface $\mathrm{SH}$ wave test is limitedly discussed.

\section{Experiment}

\subsection{Crack detection (CD) paint}

The CD paint used in this study is a mixture of a commercially-available anti-corrosion epoxy resin paint and specially-made microcapsules containing dye oil. The preparation of the microcapsules and the paint, and the painting procedure will be described below.

\subsubsection{Preparation of microcapsules}

The microcapsules were made by burying the cores, which were fine droplets of mixture of red organic dye and spindle oil, in the shells of gelatin (ThreeBond Co., Ltd.). The microcapsules were formed by the coacervation method, ${ }^{6}$ and then washed and dried. Figure 2 shows the scanning electron microscope image of the microcapsules. According to particle size measurement, the mean diameter and the most frequent diameter are $139 \mu \mathrm{m}$ and $154 \mu \mathrm{m}$ respectively.

\subsubsection{Paint and painting procedure}

As the binder of the microcapsules, an anti-corrosion epoxy resin paint (two-part, white color) was used. After sufficiently mixing the microcapsules by stirring with the blend of the resin and hardening agent, the mixture was applied by a brush around the weld toes of the specimen,

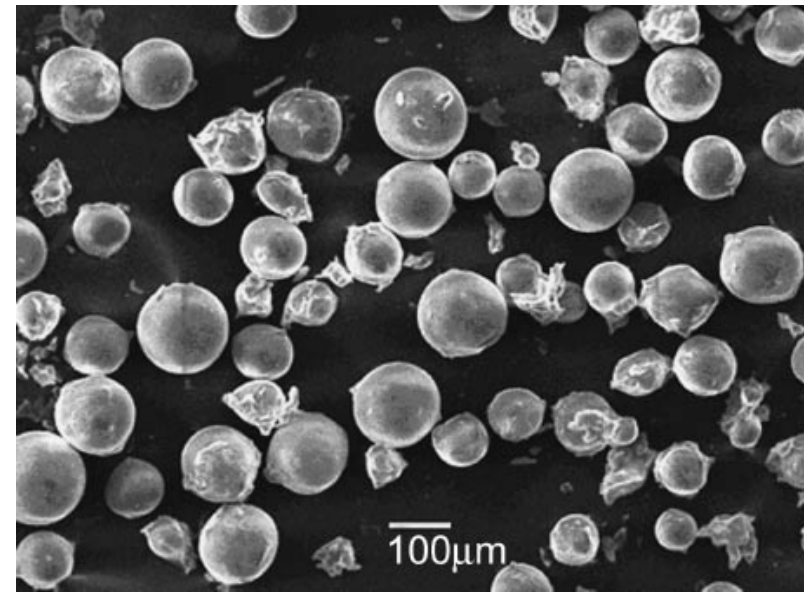

Fig. 2 SEM image of the microcapsules containing dye oil.

whose surfaces were pretreated by a wire brush.

After the paint layer containing microcapsules completely cured and hardened, the above white epoxy resin paint alone was applied as the finishing coat, by which the microcapsules distributed on the paint surface were protected and the contrast when the red color developed was increased.

\subsection{Surface $\mathrm{SH}$ wave test}

For the surface $\mathrm{SH}$ wave test, the digital ultrasonic detector UI-23 (Mitsubishi Electric Co., Ltd.), the ultrasonic surface SH wave probe 5C10x10HA90 (Japan Probe Co., Ltd.), of which the test frequency is $5 \mathrm{MHz}$, the nominal dimensions of transducer is $5 \mathrm{~mm} \times 5 \mathrm{~mm}$ and the nominal angle of refraction is 90 degree, and the glycerin paste couplant for shear waves SHN-A5 (Nichigo Acetylene Co., Ltd.) were used. Along a guide scale, which was fixed at a distance of $120 \mathrm{~mm}$ from the transverse rib surface, the reflection echo height was scanned at a pitch of $1 \mathrm{~mm}$ in the direction of the weld line. The probe was moved front and rear or right and left after application of the couplant on the test surface, and the measurement was carried out when the echo became stable (time to stabilization $=$ approximately 1 minute). Considering the feasibility and simplicity on the actual test spot, the probe was held by finger pressure without using a weight or jig for a constant pressure.

\subsection{Fatigue test of transverse rib welded joint specimen \\ 2.3.1 Material and specimen}

The material for the transverse rib welded joint specimen was a $400 \mathrm{MPa}$ class rolled steel plate for welded structures (JIS SM400B, $12 \mathrm{~mm}$ thick), with a chemical composition (mass\%) as follows: $0.13 \mathrm{C}, 0.20 \mathrm{Si}, 0.69 \mathrm{Mn}, 0.024 \mathrm{P}, 0.008 \mathrm{~S}$, remainder Fe. Similarly, the mechanical properties were: tensile strength $=432 \mathrm{MPa}$, yield stress $=283 \mathrm{MPa}$ and percentage elongation $=30.0$.

The specimen was made by attaching two transverse ribs on both surfaces of a main plate by boxing-welds. The design of the specimen is shown in Fig. 3.

\subsubsection{Fatigue test}

A load controlled fatigue test was performed using a servohydraulic fatigue tester (dynamic load capacity $=1000 \mathrm{kN}$ ). Cyclic tensile loading was applied in the longitudinal 


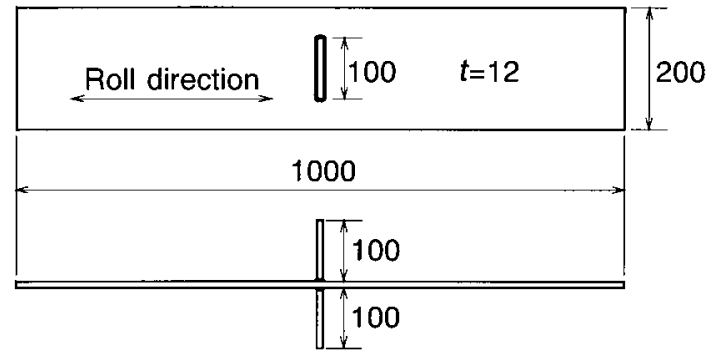

Fig. 3 Design of a transverse rib welded joint specimen.

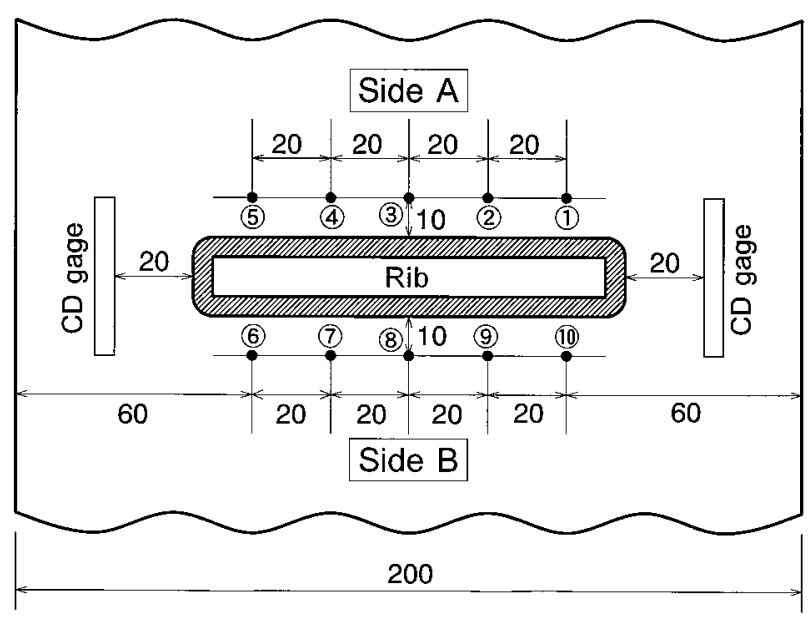

Fig. 4 Strain gauge locations.

direction of the specimen, where the load range was set to 0 $356.6 \mathrm{kN}$ (nominal stress range $\Delta \sigma_{\mathrm{n}}=148.6 \mathrm{MPa}$ ) and the test frequency was set at $2.5 \mathrm{~Hz}$.

Crack initiation life $N_{\mathrm{c}}$ was determined as the number of cycles when the strain range $10 \mathrm{~mm}$ away from the weld toe (the strain gauge locations are shown by the black dots in Fig. 4) decreased by $5 \%$ of the initial value (the $5 \%$ drop method $^{7)}$ ). This $5 \%$ decrease in the strain range corresponds to approximately 1 to $2 \mathrm{~mm}$ of crack height and 20 to $30 \mathrm{~mm}$ of crack length.

The state of crack initiation and propagation during the fatigue test was monitored by the color development in the $\mathrm{CD}$ paint, and the surface SH wave tests were carried out halting the fatigue test at proper intervals.

\section{Results and Discussions}

\subsection{Results of fatigue test}

The specimen was ruptured by a fatigue crack on side $\mathrm{A}$ in Fig. 4, which initiated and propagated along the weld toe, and the failure life, $N_{\mathrm{f}}$, was 757200 cycles. The crack initiation life, $N_{\mathrm{c}}$, which was determined by the $5 \%$ drop method mentioned in 2.3.2, was 464640 cycles, using the measurement by the No. 3 strain gauge located on the center line of side A (see Fig. 4).

On the other hand, the CD paint already started color development at $N=10000$ cycles. The states of color development at $N=163500$ and 288000 cycles are shown in Figs. 5 and 6 respectively (the color is gray in the figures, while the actual color was red). The results of the surface $\mathrm{SH}$
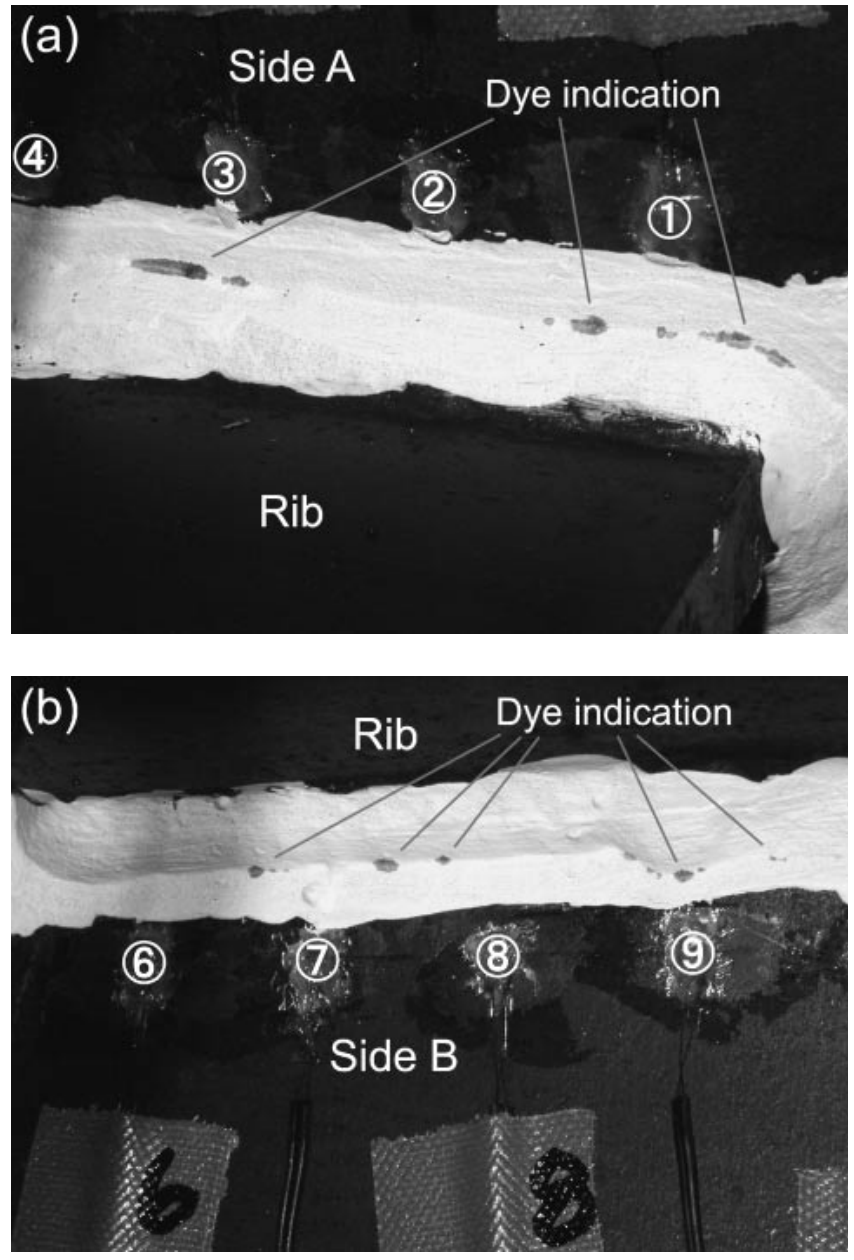

Fig. 5 Color development in the $\mathrm{CD}$ paint due to fatigue cracks $(N=$ 163500). The strain gauge spacing is $20 \mathrm{~mm}$. (a) Side A (b) Side B

wave tests and fracture surface observations, which were performed later, confirmed that the locations of color development approximately corresponded to the paths of the actual fatigue cracks. No significant decrease was observed in the strain range measured by the abovementioned No. 3 gauge at $N=288000$ cycles, and the strain range decrease at $N=336000$ cycles was only $1.3 \%$ of the initial value, proving that the $\mathrm{CD}$ paint has far higher sensitivity and accuracy in showing the timing and location of crack initiation as compared with the strain gauge method.

\subsection{Results of surface SH wave tests and color develop- ment in CD paint}

For the weld toe line on side A, where the specimen broke, the results of the surface $\mathrm{SH}$ wave tests and the state of color development in the $\mathrm{CD}$ paint were comparatively shown in Fig. 7 ( $N=480000$ cycles $)$ and Fig. $8(N=672000$ cycles $)$. The actual scanning direction was from the right to the left in the figures, and the dotted lines in the figures show the ranges of color development in the CD paint (except isolated dotted color development). Comparing Figs. 7 and 8 with the macroscopic feature of the fracture surface shown in Fig. 9, the following findings were obtained (the scale and numbers in Fig. 9 correspond to the abscissas in Figs. 7 and 8).

(1) The dark areas of the fracture surface are considered to be the marks of the dye oil, which entered into the crack 

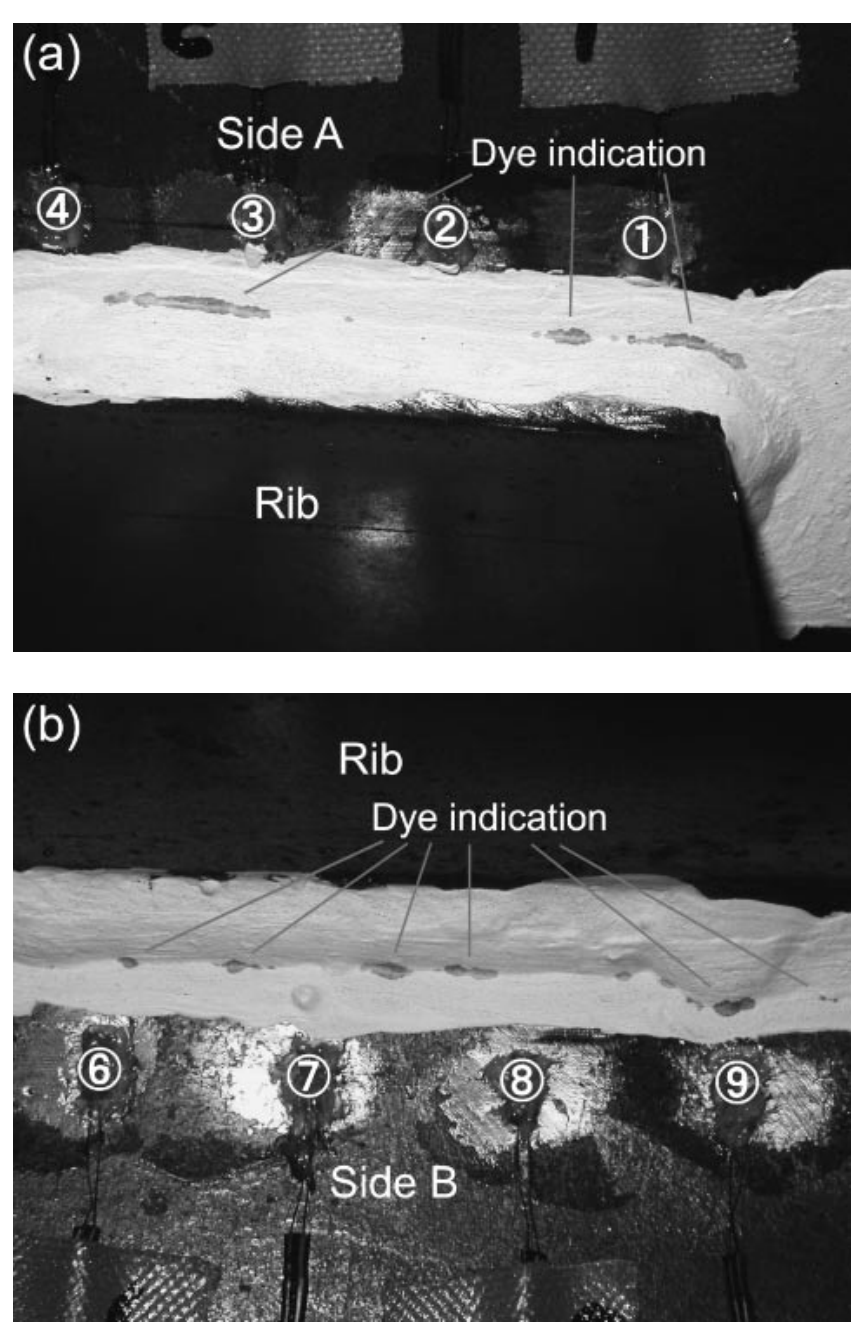

Fig. 6 Color development in the $\mathrm{CD}$ paint due to fatigue cracks $(N=$ 288000). The strain gauge spacing is $20 \mathrm{~mm}$. (a) Side A (b) Side B

pumping with the cyclic loading and then dried when the fatigue test was temporarily halted for the surface $\mathrm{SH}$ wave tests. For the left crack, the inside and darker area corresponds to $N=480000$ cycles and the outside and comparatively light area corresponds to $N=$ 672000 cycles. And it is supposed that the separate two cracks (the left one is $2.00 \mathrm{~mm}$ and the right one is $1.56 \mathrm{~mm}$ in height) at $N=480000$ cycles coalesced to a single crack (the left peak is $4.86 \mathrm{~mm}$ and the right one is $2.60 \mathrm{~mm}$ in height) at $N=672000$ cycles. That is reflected in both the color development area in the $\mathrm{CD}$ paint and the results of the surface $\mathrm{SH}$ wave tests. By comparison between the color development areas and the fracture surface patterns, it seems to be reasonable to consider that the range of color development in the CD paint approximately equals to the actual crack length.

(2) From the above fracture surface observation, it is clear that the crack near the rib center is higher than the other one near the rib end. However, the maximum echo heights were measured near the rib end both in Figs. 7 and 8 , resulting in an inverse correlation between the crack height and echo height. While this may be caused by changes in the contact state between the scanning

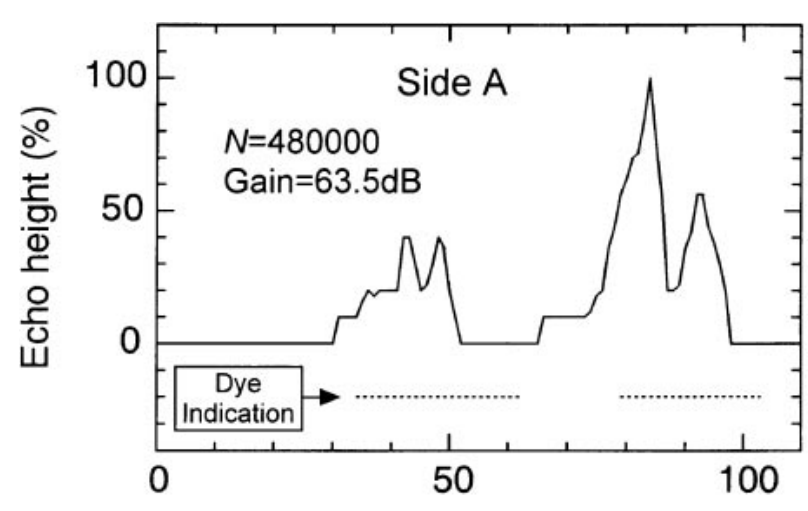

Distance from the rib end, $x / \mathrm{mm}$

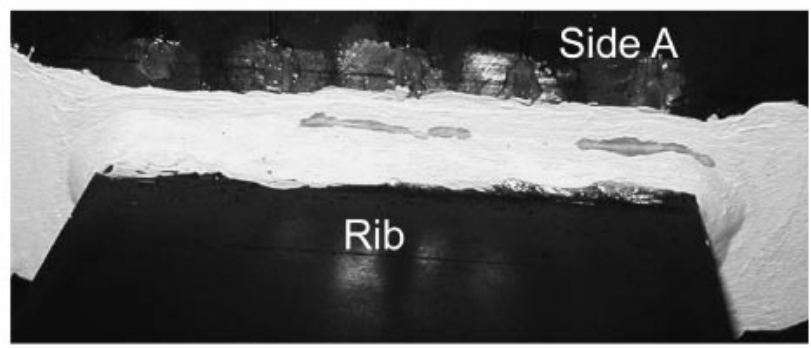

Fig. 7 Surface $\mathrm{SH}$ wave test result and color development in the CD paint ( $N=480000$, Side A). The scale of the photo approximately corresponds to the abscissa of the top figure.

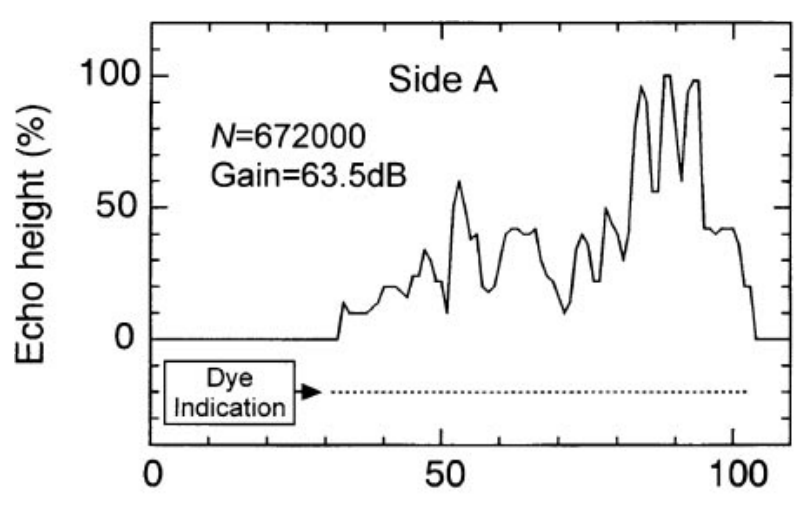

Distance from the rib end, $x / \mathrm{mm}$

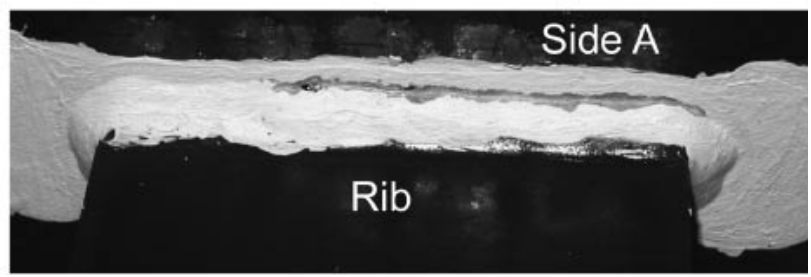

Fig. 8 Surface $\mathrm{SH}$ wave test result and color development in the CD paint ( $N=672000$, Side A). The scale of the photo approximately corresponds to the abscissa of the top figure.

probe and test surface (e.g. couplant thickness) or complicated interference between the ultrasonic waves reflected from the crack face, which actually has a three dimensional curved surface, the specific cause is not yet known. Therefore, it is difficult to estimate quantitatively the crack height from the echo height at the present state. 


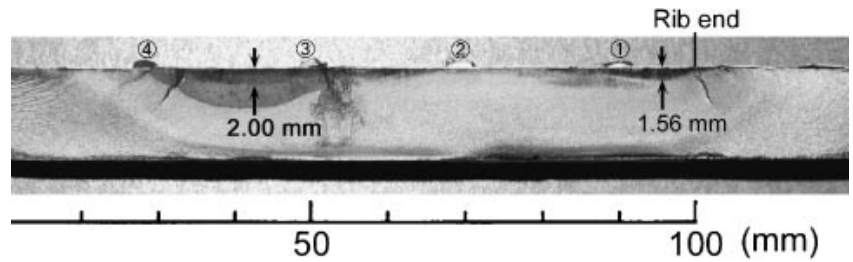

Fig. 9 Macroscopic feature of the fracture surface (Side A, $N_{\mathrm{f}}=757200$ ).

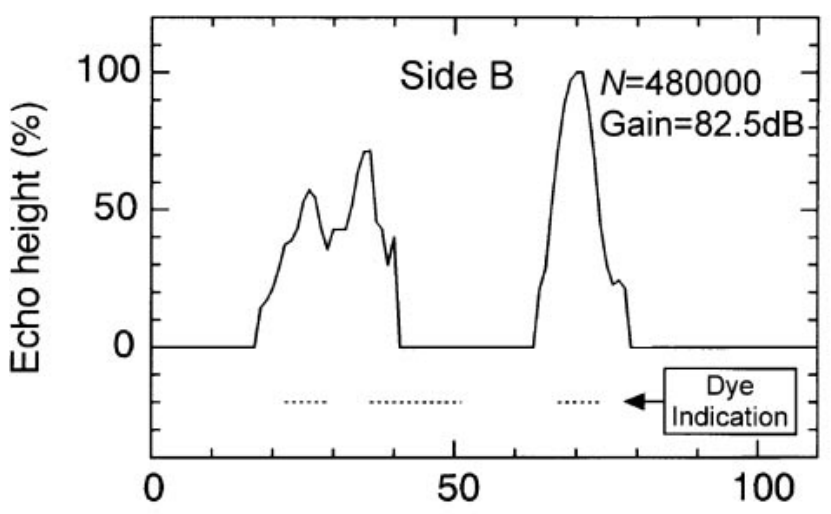

Distance from the rib end, $x / \mathrm{mm}$

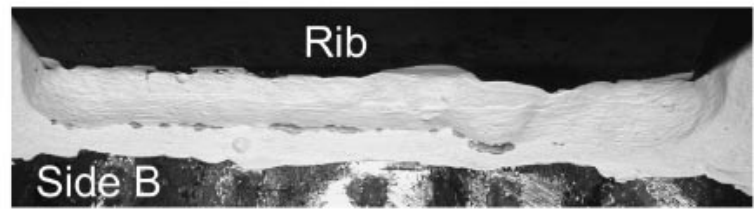

Fig. 10 Surface SH wave test result and color development in the CD paint $(N=480000$, Side B). The scale of the photo approximately corresponds to the abscissa of the top figure.

(3) Sharp fluctuation of the echo height diagram is observed in Figs. 7 and 8. This is considered to be due to the fact that the crack face near the specimen surface has irregular roughness because the surface crack propagates along the meandering weld line, and consequently the reflected ultrasonic waves interfere with each other in a complicated way.

For the weld toe line on side B, where the specimen did not broke, the results of the surface $\mathrm{SH}$ wave tests and the state of color development in the $\mathrm{CD}$ paint were comparatively shown in Fig. $10(N=480000$ cycles $)$ and Fig. $11(N=$ 672000 cycles). While the cracks were considered to be shallow and short at $N=480000$ cycles because the measuring gain was $82.5 \mathrm{~dB}$, which was approximately $20 \mathrm{~dB}$ higher than in other tests, the correlation between the surface $\mathrm{SH}$ wave test result and the color development in the $\mathrm{CD}$ paint was fairly good, proving the effectiveness of the surface SH wave test for a small crack in the early stage. Because sharp fluctuation of the echo height diagram is remarkable in Fig. 11 in the same way as Figs. 7 and 8 for side $\mathrm{A}$, it is seemed to be difficult to estimate the crack length by the ordinary $6 \mathrm{~dB}$ drop technique, in which the crack length is estimated by cutting the echo height diagram at the level of $6 \mathrm{~dB}$ lower than the peak.

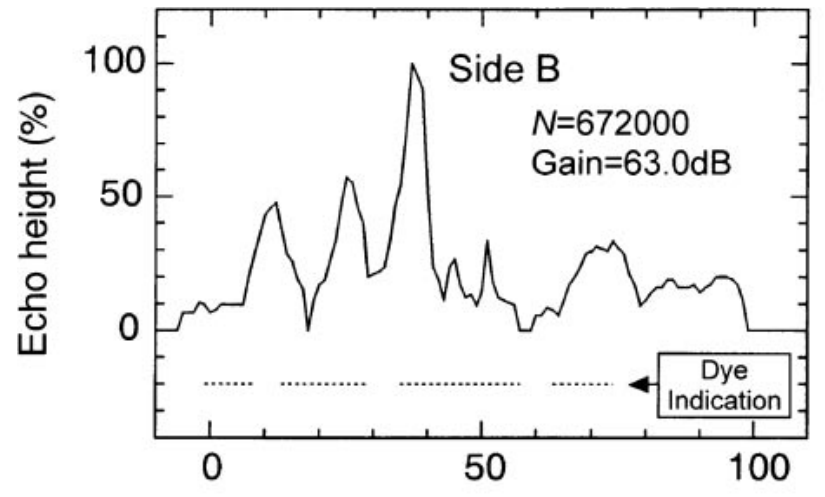

Distance from the rib end, $x / \mathrm{mm}$

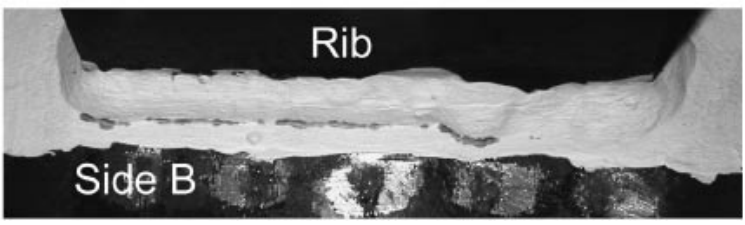

Fig. 11 Surface SH wave test result and color development in the CD paint ( $N=672000$, Side B). The scale of the photo approximately corresponds to the abscissa of the top figure.

\section{Conclusions}

In order to verify experimentally the effectiveness of the inspection technique using the $\mathrm{CD}$ paint and the surface $\mathrm{SH}$ wave test, a fatigue test of a transverse rib welded joint specimen was performed and the applicability of the combined technique to fatigue cracks at the weld toe was evaluated. Once the existence of a crack was confirmed by the surface SH wave test, the location and length of the crack can be rather easily estimated from the range of color development in the CD paint. Consequently the estimation of the crack height by the surface $\mathrm{SH}$ wave is the subject in future. The major conclusions of this work are as follows.

(1) The sensitivity of the CD paint to the fatigue cracks at the weld toe was so high that the color surely developed by the rather short cracks in the early stage, which had no effect on the measured strain range, and the color was visually recognized at ease.

(2) The surface SH wave tests gave the fairly consistent results with the color development in the $\mathrm{CD}$ paint. However, it is difficult to estimate the crack length by the ordinary $6 \mathrm{~dB}$ drop technique because the echo height diagram sharply fluctuates locally due to the interference between the echoes from the crack face.

(3) The surface SH wave test gave an inverse correlation between the actual crack height and the echo height, while the specific cause is not yet known. Therefore, it is difficult to estimate quantitatively the crack height from the echo height at the present state.

(4) The color development in the CD paint was sufficient and the surface $\mathrm{SH}$ wave tests gave rather proper results for the coalescence process of the plural cracks and for the shallow cracks in the early stage, showing the effectiveness of the combined inspection technique, in which fatigue cracks at weld toes are visually detected 
by the CD paint and then confirmed by the surface $\mathrm{SH}$ wave test.

\section{Acknowledgements}

The authors are much obliged to Dr. Yoshikazu Yokono (Pony Industry Co., Ltd.) for his kind advice and many valuable suggestions concerning the surface $\mathrm{SH}$ wave test, especially on the interference effects of ultrasonic waves reflected from a curved flaw surface.

The authors are also much obliged to Mr. Mitsuhiko Uchida, Mr. Tomio Onoguchi and Mr. Hideki Takahashi (ThreeBond Co., Ltd.) for their great contribution to the development and manufacture of the dye-containing microcapsules for the CD paint for the first time in Japan.

\section{REFERENCES}

1) SR226 committee: Shipbuilding Research Assoc. Jpn. (1996) (in Japanese).

2) R. R. Miron, W. Bilder and W. Lademan: NACE Northern Area Eastern Conf. (1999).

3) I. Takahashi, S. Akiyama, M. Ushijima, A. Takada, T. Onoguchi and M. Uchida: J. Soc. Naval Architects Jpn. Techno Marine 871 (2003) 70_ 73 (in Japanese).

4) Y. Yokono, M. Kato and K. Nishio: JOURNAL OF JSNDI 45 (1996) 136-144 (in Japanese).

5) K. Tachikawa: Welding Technology, 44 (1996) 66-71 (in Japanese).

6) T. Kondo: Microcapsule, (Kyoritsu Shuppan, Tokyo, 1985) (in Japanese).

7) SR202 committee: Research on fatigue design and quality of welded parts in offshore structures. (Shipbuilding Research Assoc. Jpn., 1991) (in Japanese). 